\title{
Half-linear Euler differential equation and its perturbations
}

\author{
Ondřej Došlý ${ }^{\otimes}$ \\ Department of Mathematics and Statistics, Masaryk University, \\ Kotlářská 2, CZ-611 37 Brno, Czech Republic \\ Appeared 11 August 2016 \\ Communicated by Tibor Krisztin
}

\begin{abstract}
We investigate oscillatory properties of a perturbed half-linear Euler differential equation. We give an alternative proof (simpler and more straightforward) of the main result of [O. Došlý, H. Funková, Abstr. Appl. Anal. 2012, Art. ID 738472] and we prove the extended version of a conjecture formulated in [O. Došlý, J. Math. Anal. Appl. 323(2006), 426-440].
\end{abstract}

Keywords: Euler half-linear differential equation, Riemann-Weber differential equation, Riccati technique, modified Riccati equation, conditional oscillation.

2010 Mathematics Subject Classification: 34C10.

\section{Introduction}

We consider the second order half-linear differential equation

$$
L(x):=\left(r(t) \Phi\left(x^{\prime}\right)\right)^{\prime}+c(t) \Phi(x)=0,
$$

where $\Phi(x)=|x|^{p-2} x$ is the odd power function and $c, r$ are continuous functions with $r(t)>0$. It is known that the linear Sturmian theory extends almost verbatim to (1.1), see [1,9], the classical Sturm-Liouville linear differential equation is the special case $p=2$ in (1.1). In particular, (1.1) can be classified as oscillatory or nonoscillatory similarly as in the linear case.

The terminology half-linear equation was introduced by Hungarian mathematicians I. Bihari and Á. Elbert and reflects the fact that the solution space of (1.1) is only homogeneous but not generally additive, so it has just one half of the properties characterizing linearity.

A "prominent position" in the half-linear oscillation theory has the Euler half-linear differential equation

$$
\left(\Phi\left(x^{\prime}\right)\right)^{\prime}+\frac{\gamma}{t^{p}} \Phi(x)=0
$$

which is a typical example of the so-called conditionally oscillatory half-linear equation. Recall that equation (1.1) with $\lambda c(t)$ instead of $c(t)$ is said to be conditionally oscillatory if there

\footnotetext{
${ }^{凶}$ Email: dosly@math.muni.cz
} 
exists a constant $\lambda_{0}$ (the so-called oscillation constant) such that (1.1) is oscillatory for $\lambda>\lambda_{0}$ and nonoscillatory for $\lambda<\lambda_{0}$. Oscillation constant of Euler equation (1.2) is $\gamma_{p}:=\left(\frac{p-1}{p}\right)^{p}$.

The conditional oscillation of (1.2) together with the Sturmian comparison theorem immediately imply the Kneser type (non)oscillation criteria for (1.1) with $r(t)=1$, namely, this equation is oscillatory if

and it is nonoscillatory if

$$
\liminf _{t \rightarrow \infty} t^{p} c(t)>\gamma_{p}
$$

$$
\limsup _{t \rightarrow \infty} t^{p} c(t)<\gamma_{p}
$$

These (non)oscillation conditions show that Euler equation (1.2) with the oscillation constant $\gamma_{p}$ is a kind of borderline between oscillation and nonoscillation of half-linear equations and suggests the investigation of the limiting case $\lim _{t \rightarrow \infty} t^{p} c(t)=\gamma_{p}$.

In our paper we investigate the influence of perturbations of the critical Euler equation (i.e., of (1.2) with $\gamma=\gamma_{p}$ ) on the oscillation behavior of perturbed equations. We are motivated by the recent papers $[6-8,10,12,15,17]$ where a similar problem was investigated. In the general setting, we consider the half-linear equation

$$
\left[(r(t)+\hat{r}(t)) \Phi\left(x^{\prime}\right)\right]^{\prime}+(c(t)+\hat{c}(t)) \Phi(x)=0
$$

as a perturbation on the nonoscillatory equation (1.1). The situation when a perturbation is also allowed in the differential term was treated in the linear case in [16]. An extension of the results of [16] to $(1.3)$ is given in $[4,5,15]$. In [6], the differential equation

$$
\left[\left(1+\sum_{j=1}^{n} \frac{\alpha_{j}}{\log _{j}^{2}(t)}\right) \Phi\left(x^{\prime}\right)\right]^{\prime}+\frac{1}{t^{p}}\left(\gamma_{p}+\sum_{j=1}^{n} \frac{\beta_{j}}{\log _{j}^{2}(t)}\right) \Phi(x)=0
$$

is considered, where the notations

$$
\log _{k}(t)=\log _{k-1}(\log t), \quad \log _{1}(t)=\log t, \quad \log _{k}(t)=\prod_{j=1}^{k} \log _{j}(t)
$$

are used. It was shown that oscillation of (1.4) depends on the value of the constants $\beta_{j}-\gamma_{p} \alpha_{j}-\mu_{p}$, where $\mu_{p}=\frac{1}{2}\left(\frac{p-1}{p}\right)^{p-1}$. This statement is proved in [6] using the transformation theory of the so-called modified Riccati equation associated with (1.4).

As one of the main results of our paper we offer an alternative proof which is simpler and more straightforward than that given in [6]. As another main result, we prove a conjecture presented in [2] which concerns perturbations of the half-linear Riemann-Weber differential equation (sometime also called Euler-Weber equation).

\section{Preliminaries}

In the oscillation theory of (1.1), an important role is played by the associated Riccati type differential equation

$$
R[w]:=w^{\prime}+c(t)+(p-1) r^{1-q}(t)|w|^{q}=0, \quad \frac{1}{p}+\frac{1}{q}=1,
$$

related to (1.1) by the substitution $w=r \Phi\left(x^{\prime} / x\right)$. More precisely, the following statement holds (see [9, p. 50]). 
Proposition 2.1. Equation (1.1) is nonoscillatory if and only if there exists a solution of Riccati equation (2.1) which is defined on some interval $[T, \infty)$ (the so-called proper solution).

We will also need the so-called modified Riccati equation. Let $h$ be a differentiable function and let

$$
v(t)=h^{p}(t) w(t)-G(t), \quad G(t):=r(t) h(t) \Phi\left(h^{\prime}(t)\right),
$$

where $w$ is a solution of (2.1). Then $v$ is a solution of the modified Riccati equation

$$
\mathcal{R}[v]:=v^{\prime}+C(t)+(p-1) r^{1-q}(t) h^{-q}(t) H(v, G(t))=0,
$$

where

$$
H(v, G)=|v+G|^{q}-q \Phi^{-1}(G) v-|G|^{q}=0,
$$

$\Phi^{-1}(x)=|x|^{q-2} x$ being the inverse function of $\Phi$, and

$$
C(t)=h(t) L(h(t))=h(t)\left[\left(r(t) \Phi\left(h^{\prime}(t)\right)+c(t) \Phi(h(t))\right] .\right.
$$

The modified Riccati operator $\mathcal{R}$ is related to (1.1) and (2.1) by the identities

$$
x L(x)=x^{p} R[w]=\mathcal{R}[v],
$$

where $w=r \Phi\left(x^{\prime} / x\right)$ and $v=x^{p} w-G, G=r x \Phi\left(x^{\prime}\right)$, see e.g. [3].

The basic paper dealing with perturbations of the Euler equation is [12]. In that paper, following the linear case, see [14, Chapter XI], the generalized Riemann-Weber half-linear differential equation

$$
\left(\Phi\left(x^{\prime}\right)\right)^{\prime}+\frac{1}{t^{p}}\left[\gamma_{p}+\sum_{j=1}^{n} \frac{\beta_{j}}{\log _{j}^{2}(t)}\right] \Phi(x)=0
$$

is considered. It was shown that if

$$
\beta_{j}=\mu_{p}:=\frac{1}{2}\left(\frac{p-1}{p}\right)^{p-1}, \quad j=1, \ldots, n-1,
$$

then (3.13) is oscillatory if and only if $\beta_{n}>\mu_{p}$. This result was extended in [6], where oscillatory properties of (1.4) were investigated, i.e., a perturbation of (1.2) with $\gamma=\gamma_{p}$ was also allowed in the term containing $\Phi\left(x^{\prime}\right)$. It was shown that if $\beta_{j}-\gamma_{p} \alpha_{j}=\mu_{p}, j=1, \ldots, n-1$, then (1.4) is oscillatory if and only if $\beta_{n}-\gamma_{p} \alpha_{n}>\mu_{p}$.

The next part is devoted to the existence of proper solutions of the modified Riccati equation (2.3).

Proposition 2.2. ([6, Proposition 2.2]). Let $h$ be a continuously differentiable function such that $h^{\prime}(t) \neq 0$ for large $t$. We denote $R(t)=r(t) h^{2}(t)\left|h^{\prime}(t)\right|^{p-2}$. Suppose that

$$
\int^{\infty} R^{-1}(t) d t=\infty
$$

holds and that

$$
\liminf _{t \rightarrow \infty}|G(t)|>0
$$

where $G$ is given by (2.2). 
(i) If $C(t) \leq 0$ for large $t$, where $C$ is given by (2.4), then (2.3) possesses a proper solution, i.e., $a$ solution which exists on some interval $[T, \infty)$.

(ii) Let $C(t) \geq 0$ for large $t$ and $\int^{\infty} C(t) d t<\infty$. If

$$
\liminf _{t \rightarrow \infty}\left(\int^{t} R^{-1}(s) d s\right)\left(\int_{t}^{\infty} C(s) d s\right)>\frac{1}{2 q^{\prime}}
$$

then (2.3) possesses no proper solution, i.e., for any solution $v$ of (2.3) and any $T \in \mathbb{R}$ there exists $T_{1}>T$ such that $v\left(T_{1}-\right)=-\infty$.

(iii) If $\int^{\infty} C(t) d t$ is convergent and

$$
\begin{aligned}
& \limsup _{t \rightarrow \infty}\left(\int^{t} R^{-1}(s) d s\right)\left(\int_{t}^{\infty} C(s) d s\right)<\frac{1}{2 q^{\prime}} \\
& \liminf _{t \rightarrow \infty}\left(\int^{t} R^{-1}(s) d s\right)\left(\int_{t}^{\infty} C(s) d s\right)>-\frac{3}{2 q},
\end{aligned}
$$

then (2.3) has a proper solution.

\section{Oscillation and nonoscillation criteria}

As an immediate consequence of propositions from the previous sections we have the following oscillation criteria for (1.3) viewed as a perturbation of nonoscillatory equation (1.1). We denote

$$
\hat{L}(x):=\left(\hat{r}(t) \Phi\left(x^{\prime}\right)\right)^{\prime}+\hat{c}(t) \Phi(x) .
$$

Associated with equation (1.3) the Riccati equation is

$$
w^{\prime}+c(t)+\hat{c}(t)+(p-1)(r(t)+\hat{r}(t))^{1-q}|w|^{q}=0
$$

and the modified Riccati equation

$$
v^{\prime}+C(t)+\hat{C}(t)+(p-1)(r(t)+\hat{r}(t))^{1-q} h^{-q}(t) H(v, \Omega)=0,
$$

with

$$
\Omega(t):=(r(t)+\hat{r}(t)) h(t) \Phi\left(h^{\prime}(t)\right),
$$

$C$ given by (2.4), and

$$
\hat{C}(t)=h(t) \hat{L}(h(t))=h(t)\left[\left(\hat{r}(t) \Phi\left(h^{\prime}(t)\right)+\hat{c}(t) \Phi(h(t)) .\right.\right.
$$

Theorem 3.1. Let $h$ be a continuously differentiable function such that $h^{\prime}(t) \neq 0$ for large $t$. Suppose that (2.5) holds with $R$ defined now as

$$
R(t)=(r(t)+\hat{r}(t)) h^{2}(t)\left|h^{\prime}(t)\right|^{p-2}
$$

and

$$
\liminf _{t \rightarrow \infty}|\Omega(t)|>0
$$


(i) Suppose that $C(t)+\hat{C}(t) \geq 0$ for large $t$ and $\int^{\infty}[C(t)+\hat{C}(t)] d t<\infty$, where $C, \hat{C}$ are given by (2.4) and (3.3). If

$$
\liminf _{t \rightarrow \infty}\left(\int^{t} R^{-1}(s) d s\right)\left(\int_{t}^{\infty}(C(s)+\hat{C}(s)) d s\right)>\frac{1}{2 q},
$$

then (1.3) is oscillatory.

(ii) If the integral $\int^{\infty}[C(t)+\hat{C}(t)] d t$ is convergent and

$$
\begin{gathered}
\limsup _{t \rightarrow \infty}\left(\int^{t} R^{-1}(s) d s\right)\left(\int_{t}^{\infty}[C(s)+\hat{C}(s)] d s\right)<\frac{1}{2 q}, \\
\liminf _{t \rightarrow \infty}\left(\int^{t} R^{-1}(s) d s\right)\left(\int_{t}^{\infty}[C(s)+\hat{C}(s)] d s\right)>-\frac{3}{2 q},
\end{gathered}
$$

then (1.3) is nonoscillatory.

Proof. The proof immediately follows from Proposition 2.2. First, suppose that (3.5) holds, and, by contradiction, that (1.3) is nonoscillatory. Then (3.1) has a proper solution $w$ and $v=h^{p} w-\Omega$ is a proper solution of (3.2), a contradiction. Conversely, suppose that (3.6) holds. Then (3.2) possesses a proper solution $v$ and $w=h^{-p}(v+\Omega)$ is a proper solution of (3.1) which means, by Proposition 2.1, that (1.3) is nonoscillatory.

Now we apply the previous result to the perturbed Euler equation (1.4). Recall that equation (1.2) with $\gamma=\gamma_{p}$ is nonoscillatory and one of its solutions is $x(t)=t^{(p-1) / p}$. Any solution linearly independent of this function asymptotically behaves as the function $x(t)=C t^{(p-1) / p} \log ^{1 / p} t, C \in \mathbb{R}$, see [11].

If $\alpha_{j}=0$ and $\beta_{j}=\mu_{p}, j=1, \ldots, n$ in (1.4), it is shown in [12] that this equation has a solution asymptotically equivalent to $x(t)=t^{(p-1) / p} \log _{n}^{1 / p}(t)$. The function of this form is used in the modified Riccati substitution in the main part of the proof of the next statement. As we have already mentioned earlier, this statement is proved in [6] using relatively awkward transformation theory of modified Riccati equation. Our proof here is technically substantially easier.

Theorem 3.2. Consider equation (1.4).

(i) If $\beta_{1}-\gamma_{p} \alpha_{1}>\mu_{p}$, then (1.4) is oscillatory and if $\beta_{1}-\gamma_{p} \alpha_{1}<\mu_{p}$ then (1.4) is nonoscillatory.

(ii) If $\beta_{1}-\gamma_{p} \alpha_{1}=\mu_{p}$, then (1.4) is oscillatory if $\beta_{2}-\gamma_{p} \alpha_{2}>\mu_{p}$ and it is nonoscillatory if $\beta_{2}-\gamma_{p} \alpha_{2}<\mu_{p}$.

(iii) If $\beta_{2}-\gamma_{p} \alpha_{2}=\mu_{p}$, then (1.4) is oscillatory if $\beta_{3}-\gamma_{p} \alpha_{3}>\mu_{p}$ and it is nonoscillatory if $\beta_{3}-\gamma_{p} \alpha_{3}<\mu_{p}$.

(n) If $\beta_{j}-\gamma_{p} \alpha_{j}=\mu_{p}$ for $j=1, \ldots, n-1$, then (1.4) is oscillatory if $\beta_{n}-\gamma_{p} \alpha_{n}>\mu_{p}$ and it is nonoscillatory if $\beta_{n}-\gamma_{p} \alpha_{n}<\mu_{p}$.

$(n+1)$ If $\beta_{j}-\gamma_{p} \alpha_{j}=\mu_{p}$ for all $j=1, \ldots, n$, then (1.4) is nonoscillatory. 
Proof. Observe that we have for $n \geq 2$ and large $t$ the obvious inequalities

$$
\log _{n}(t)>\cdots>\log _{1}(t)=\log t>\cdots>\log _{n}(t) .
$$

We also have $\left(\log _{n}(t)\right)^{\prime}=1 /\left(t \log _{n-1}(t)\right)$ and

$$
\left(\log _{n}(t)\right)^{\prime}=\frac{\log _{n}(t)}{t}\left(\frac{1}{\log t}+\frac{1}{\log _{2}(t)}+\cdots+\frac{1}{\log _{n}(t)}\right) .
$$

First, let $\beta_{1}-\gamma_{p} \alpha_{1} \neq \mu_{p}$. In this case we take $h(t)=t^{(p-1) / p}$. Then, using the notation $\Gamma_{p}:=\left(\frac{p-1}{p}\right)^{p-1}$ and (3.7), (3.8)

$$
\begin{aligned}
C+\hat{C} & =h\left[\left(1+\sum_{j=1}^{n} \frac{\alpha_{j}}{\log _{j}^{2}(t)}\right) \Phi\left(h^{\prime}\right)\right]^{\prime}+\frac{h^{p}}{t^{p}}\left[\gamma_{p}+\sum_{j=1}^{n} \frac{\beta_{j}}{\log _{j}^{2}(t)}\right] \\
& =t^{\frac{p-1}{p}}\left[\Gamma_{p}\left(1+\sum_{j=1}^{n} \frac{\alpha_{j}}{\log _{j}^{2}(t)}\right) t^{-\frac{p-1}{p}}\right]^{\prime}+\frac{1}{t}\left[\gamma_{p}+\sum_{j=1}^{n} \frac{\beta_{j}}{\log _{j}^{2}(t)}\right] \\
& =\frac{\beta_{1}-\gamma_{p} \alpha_{1}}{t \log ^{2} t}+o\left(t^{-1} \log ^{-2} t\right)
\end{aligned}
$$

as $t \rightarrow \infty$. We have for $h(t)=t^{(p-1) / p}$

$$
R(t)=\left(1+\sum_{j=1}^{n} \frac{\alpha_{j}}{\log _{j}^{2}(t)}\right) h^{2}(t)\left|h^{\prime}(t)\right|^{p-2}=\left(\frac{p-1}{p}\right)^{p-2} t(1+o(1))
$$

and

$$
\Omega(t)=\left(1+\sum_{j=1}^{n} \frac{\alpha_{j}}{\log _{j}^{2}(t)}\right) h(t) \Phi\left(h^{\prime}(t)\right)=\Gamma_{p}(1+o(1))
$$

as $t \rightarrow \infty$. Hence

$$
\int^{t} R^{-1}(s) d s=\left(\frac{p-1}{p}\right)^{2-p} \log t(1+o(1))
$$

and substituting into (3.5) and (3.6) we have oscillation if

$$
\beta_{1}-\gamma_{p} \alpha_{1}>\frac{1}{2 q}\left(\frac{p-1}{p}\right)^{p-2}=\frac{1}{2}\left(\frac{p-1}{p}\right)^{p-1}=\mu_{p}
$$

and nonoscillation if $\beta_{1}-\gamma_{p} \alpha_{1}<\mu_{p}$. Note that the second limit in (3.6) is not needed in our case since the term $\left(\beta_{1}-\gamma_{p} \alpha_{1}\right) t^{-1} \log ^{-2} t$ dominates other terms in $C+\hat{C}$ so this term is eventually of one sign. If it is negative, the statement follows from (i) of Proposition 2.2.

Now, let $n \in \mathbb{N}, \beta_{j}-\gamma_{p} \alpha_{j}=\mu_{p}, j=1, \ldots, n-1$, and $\beta_{n}-\gamma_{p} \alpha_{n} \neq \mu_{p}$. Let $h(t)=$ $t^{(p-1) / p} \log _{n-1}^{1 / p}(t)$. Then using (3.8),

$h^{\prime}(t)=\frac{p-1}{p} t^{-\frac{1}{p}} \log _{n-1}^{\frac{1}{p}}(t)\left(1+\frac{1}{(p-1) \log t}+\frac{1}{(p-1) \log _{2}(t)}+\cdots+\frac{1}{(p-1) \log _{n-1}(t)}\right)$

and

$$
\begin{aligned}
\Phi\left(h^{\prime}\right)= & \Gamma_{p} t^{-\frac{p-1}{p}} \log _{n-1}^{\frac{p-1}{p}}(t) \\
& \times\left(1+\frac{1}{(p-1) \log t}+\frac{1}{(p-1) \log _{2}(t)}+\cdots+\frac{1}{(p-1) \log _{n-1}(t)}\right)^{p-1} .
\end{aligned}
$$


Denote $F=\left(\Phi\left(h^{\prime}\right)\right)^{\prime}$. Then

$$
\begin{aligned}
& F=t^{-2+\frac{1}{p}} \log _{n-1}^{\frac{p-1}{p}} t\left(1+\frac{1}{(p-1) \log t}+\frac{1}{(p-1) \log _{2}(t)}+\cdots+\frac{1}{(p-1) \log _{n-1}(t)}\right)^{p-2} \\
& \times\{-\gamma_{p}\left(1+\frac{1}{(p-1) \log t}+\frac{1}{(p-1) \log _{2}(t)}+\cdots+\frac{1}{(p-1) \log _{n-1}(t)}\right) \\
&+\gamma_{p}\left(\frac{1}{\log t}+\frac{1}{\log _{2}(t)}+\cdots+\frac{1}{\log _{n-1}(t)}\right) \\
& \times\left(1+\frac{1}{(p-1) \log t}+\frac{1}{(p-1) \log _{2}(t)}+\cdots+\frac{1}{(p-1) \log _{n-1}(t)}\right) \\
&-\Gamma_{p}\left[\frac{1}{\log ^{2} t}+\frac{1}{\log (t)}\left(\frac{1}{\log t}+\frac{1}{\log _{2}(t)}\right)+\ldots\right. \\
&\left.\left.+\frac{1}{\log _{n-1}(t)}\left(\frac{1}{\log t}+\cdots+\frac{1}{\log _{n-1}(t)}\right)\right]\right\} \\
&=t+\frac{1}{p} \log _{n-1}^{\frac{p-1}{p}(t)}\left(1+\frac{1}{\log t}+\frac{1}{\log _{2}(t)}+\cdots+\frac{1}{\log _{n-1}(t)}\right)^{p-2} \\
& \times-\gamma_{p}+\frac{\gamma_{p}(p-2)}{p-1}\left(\frac{1}{\log t}+\cdots+\frac{1}{\log _{n-1}(t)}\right)-\gamma_{p}\left(\frac{1}{\log ^{2} t}+\cdots+\frac{1}{\log _{n-1}^{2}(t)}\right) \\
&\left.-\frac{\Gamma_{p}(p-2)}{p} \sum_{1 \leq i<j \leq n-1} \frac{1}{\log _{i}(t) \log _{j}(t)}\right\} \cdot
\end{aligned}
$$

Denote by $A$ the expression in $\{\cdot\}$ in the last computation and let

$$
B:=\left(1+\frac{1}{(p-1) \log t}+\frac{1}{(p-1) \log _{2}(t)}+\cdots+\frac{1}{(p-1) \log _{n-1}(t)}\right)^{p-2} .
$$

Then using (3.7)

$$
\begin{aligned}
B=1 & +\frac{p-2}{p-1}\left(\frac{1}{\log t}+\cdots+\frac{1}{\log _{n-1}(t)}\right)+\frac{(p-2)(p-3)}{2(p-1)^{2}}\left(\frac{1}{\log t}+\cdots+\frac{1}{\log _{n-1}(t)}\right)^{2} \\
& +O\left(\log ^{-3} t\right)
\end{aligned}
$$

and

$$
\begin{aligned}
A \cdot B= & -\gamma_{p}+\frac{\gamma_{p}(p-2)}{p-1}\left(\frac{1}{\log t}+\cdots+\frac{1}{\log _{n-1}(t)}\right) \\
& -\frac{\gamma_{p}(p-2)}{p-1}\left(\frac{1}{\log t}+\cdots+\frac{1}{\log _{n-1}(t)}\right) \\
& -\gamma_{p}\left(\frac{1}{\log ^{2} t}+\cdots+\frac{1}{\log _{n}^{2}(t)}\right)+\frac{\gamma_{p}(p-2)^{2}}{(p-1)^{2}}\left(\frac{1}{\log t}+\cdots+\frac{1}{\log _{n-1}(t)}\right)^{2} \\
& -\frac{\gamma_{p}(p-2)(p-3)}{2(p-1)^{2}}\left(\frac{1}{\log t}+\cdots+\frac{1}{\log _{n-1}(t)}\right)^{2} \\
& -\frac{\Gamma_{p}(p-2)}{p} \sum_{1 \leq i<j \leq n-1} \frac{1}{\log _{i}(t) \log _{j}(t)} \\
& +O\left(\log ^{-3} t\right)
\end{aligned}
$$




$$
\begin{aligned}
= & -\gamma_{p}+\gamma_{p}\left(-1 \frac{(p-2)^{2}}{(p-1)^{2}}-\frac{(p-2)(p-3)}{2(p-1)^{2}}\right)\left(\frac{1}{\log ^{2} t}+\cdots+\frac{1}{\log _{n-1}^{2}(t)}\right) \\
& +\gamma_{p}\left(\frac{2(p-2)^{2}}{(p-1)^{2}}-\frac{(p-2)(p-3)}{(p-1)^{2}}\right) \sum_{1 \leq i<j \leq n-1} \frac{1}{\log _{i}(t) \log _{j}(t)} \\
& -\frac{\Gamma_{p}(p-2)}{p} \sum_{1 \leq i<j \leq n-1} \frac{1}{\log _{i}(t) \log _{j}(t)}+O\left(\log ^{-3} t\right) \\
= & -\gamma_{p}-\left(\frac{p-1}{p}\right)^{p} \frac{p}{2(p-1)} \sum_{j=1}^{n-1} \frac{1}{\log _{j}^{2}(t)} \\
& +(p-2)\left(\frac{\gamma_{p}}{p-1}-\frac{\Gamma_{p}}{p}\right) \sum_{1 \leq i<j \leq n-1} \frac{1}{\log _{i}(t) \log _{j}(t)}+O\left(\log ^{-3} t\right) \\
= & -\gamma_{p}-\mu_{p} \sum_{j=1}^{n-1} \frac{1}{\log _{j}^{2}(t)}+O\left(\log ^{-3} t\right)
\end{aligned}
$$

as $t \rightarrow \infty$. Hence

$$
h\left(\Phi\left(h^{\prime}\right)\right)^{\prime}=-\frac{\log _{n-1}(t)}{t}\left(\gamma_{p}+\mu_{p} \sum_{j=1}^{n-1} \frac{1}{\log _{j}^{2}(t)}+O\left(\log ^{-3} t\right)\right) .
$$

Next we estimate the term $\log _{n-1}(t) /\left(t \log ^{3} t\right)$. To do this, observe that for $\varepsilon \in(0,1)$ we have for $n \geq 2$

$$
\lim _{t \rightarrow \infty} \frac{\log _{n-1}(t)}{\log ^{1+\varepsilon} t}=0,
$$

as can be shown by a direct computation and hence for large $t$

$$
\int_{t}^{\infty} \frac{\log _{n-1}(s)}{s \log ^{3} s} d s<\int_{t}^{\infty} \frac{1}{s \log ^{2-\varepsilon}} d s=\frac{\text { const }}{\log ^{1-\varepsilon} t} .
$$

Consequently, for any integer $k \geq 2$

$$
\lim _{t \rightarrow \infty} \log _{k}(t) \int_{t}^{\infty} \frac{\log _{n-1}(s)}{s \log ^{3} s} d s=0 .
$$

This shows (see below) that we can neglect the terms $O\left(\log _{n-1}(t) /\left(t \log ^{3} t\right)\right)$ in some of the next computations.

Using the previous computations

$$
\begin{aligned}
{\left[\frac{\Phi\left(h^{\prime}\right)}{\log _{j}^{2}(t)}\right]^{\prime} } & =-\frac{2 \Gamma_{p} \log _{n-1}^{\frac{p-1}{p}}(t)}{t^{2-\frac{1}{p}} \log t \log _{j}^{2}(t)}(1+o(1))+\frac{\left(\Phi\left(h^{\prime}\right)\right)^{\prime}}{\log _{j}^{2}(t)} \\
& =\frac{\log _{n-1}^{\frac{p-1}{p}}(t)}{t^{2-\frac{1}{p}}}\left(-\gamma_{p}-\mu_{p} \sum_{j=1}^{n-1} \frac{1}{\log _{j}^{2}(t)}+O\left(\log ^{-3} t\right)\right)
\end{aligned}
$$

and so 


$$
h\left(\frac{\alpha_{j}}{\log _{j}^{2}(t)} \Phi\left(h^{\prime}\right)\right)^{\prime}=-\alpha_{j} \gamma_{p} \frac{\log _{n-1}(t)}{t \log _{j}^{2}(t)}+O\left(\log _{n-1}(t) t^{-1} \log ^{-3} t\right)
$$

Denote

$$
L_{E}(h):=\left[\left(1+\sum_{j=1}^{n} \frac{\alpha_{j}}{\log _{j}^{2}(t)}\right) \Phi\left(h^{\prime}\right)\right]^{\prime}+\frac{1}{t^{p}}\left[\gamma_{p}+\sum_{j=1}^{n} \frac{\beta_{j}}{\log _{j}^{2}(t)}\right] \Phi(h) .
$$

Then

$$
\begin{aligned}
h L_{E}(h)= & -\frac{\gamma_{p} \log _{n-1}(t)}{t}-\sum_{j=1}^{n-1} \frac{\left(\mu_{p}+\alpha_{j} \gamma_{p}\right) \log _{n-1}(t)}{t \log _{j}^{2}(t)}-\frac{\gamma_{p} \alpha_{n} \log _{n-1}(t)}{t \log _{n}^{2}(t)} \\
& +\frac{\gamma_{p} \log _{n-1}(t)}{t}+\sum_{j=1}^{n} \frac{\beta_{j} \log _{n-1}(t)}{t \log _{j}^{2}(t)}+O\left(\log _{n-1}(t) t^{-1} \log ^{-3} t\right) \\
= & \sum_{j=1}^{n-1} \frac{\left(\beta_{j}-\mu_{p}-\alpha_{j} \gamma_{p}\right) \log _{n-1}(t)}{t \log _{j}^{2}(t)}+\frac{\left(\beta_{n}-\gamma_{p} \alpha_{n}\right) \log _{n-1}(t)}{t \log _{n}^{2}(t)} \\
= & \frac{\beta_{n}-\gamma_{p} \alpha_{n}}{t \log _{n-1}(t) \log _{n}^{2}(t)}+O\left(\log _{n-1}(t) t^{-1} \log ^{-3} t\right),
\end{aligned}
$$

which means, in view of (3.9), that

$$
\int_{t}^{\infty}[C(s)+\hat{C}(s)] d s=\int_{t}^{\infty} h(s) L_{E}(h(s)) d s=(1+o(1)) \frac{\beta_{n}-\gamma_{p} \alpha_{n}}{\log _{n}(t)} .
$$

We finish this part of the proof by applying Theorem 3.1. We have by a direct computation for $h(t)=t^{(p-1) / p} \log _{n-1}^{1 / p}(t)$ and $\hat{r}(t)=\sum_{j=1}^{n} \frac{\alpha_{j}}{\log _{j}^{2}(t)}$

$$
(1+\hat{r}(t)) h^{2}(t)\left|h^{\prime}(t)\right|^{p-2}=\left(\frac{p-1}{p}\right)^{p-2} t \log _{n-1}(t)(1+o(1)),
$$

i.e.,

$$
\int^{t} R^{-1}(s) d s=(1+o(1))\left(\frac{p-1}{p}\right)^{2-p} \log _{n}(t)
$$

Consequently,

$$
\left.\lim _{t \rightarrow \infty}\left(\int^{t} R^{-1}(s) d s\right)\left(\int_{t}^{\infty}[C(s)+\hat{C}(s)] d s\right]\right)=\left(\frac{p-1}{p}\right)^{2-p}\left(\beta_{n}-\gamma_{p} \alpha_{n}\right) .
$$

Since $\frac{1}{2 q}\left(\frac{p-1}{p}\right)^{p-2}=\mu_{p}$, the statement of the theorem follows from Theorem 3.1.

If the equality $\beta_{j}-\gamma_{p} \alpha_{j}=\mu_{p}$ holds for $j=1, \ldots, k-1$ and $\beta_{k}-\gamma_{p} \alpha_{k}=\mu_{p}$ for some index $k \leq n-1$, we use the transformation function $h(t)=t^{(p-1) / p} \log _{k-1}^{1 / p}(t)$. A computation, quite analogous to the previous one with $n=k$, gives

$$
\begin{aligned}
h L_{E}(h)= & \frac{\left(\beta_{k}-\gamma_{p} \alpha_{k}\right) \log _{k-1}(t)}{t \log _{k}^{2}(t)}+\sum_{j=k+1}^{n} \frac{\left(\beta_{j}-\gamma_{p} \alpha_{j}\right) \log _{k-1}(t)}{t \log _{j}^{2}(t)} \\
& +O\left(\log _{k-1}(t) t^{-1} \log ^{-3} t\right)
\end{aligned}
$$


as $t \rightarrow \infty$. By a direct computation similar to that proving (3.9) we find that

$$
\lim _{t \rightarrow \infty} \log _{k}(t) \int_{t}^{\infty}\left(\sum_{j=k+1}^{n} \frac{\left(\beta_{j}-\gamma_{p} \alpha_{j}\right) \log _{k-1}(s)}{s \log _{j}^{2}(s)}\right) d s=0 .
$$

Hence the term $\left(\beta_{k}-\gamma_{p} \alpha_{k}\right) /\left(t \log _{k-1}(t) \log _{k}^{2}(t)\right)$ dominates other ones in $h L_{E}(h)$ and the statement follows from Theorem 3.1 since

$$
\int_{t}^{\infty} \frac{1}{s \log _{k-1}(s) \log _{k}^{2}(s)} d s=\frac{1}{\log _{k}(t)}
$$

Finally, suppose that $\beta_{j}-\gamma_{p} \alpha_{j}=\mu_{p}$ for all $j=1, \ldots, n$. In this case we use the transformation function $h(t)=t^{(p-1) / p} \log _{n}^{1 / p}(t)$ with the result

$$
h L_{E}(h)=O\left(\log _{n}(t) t^{-1} \log ^{-3} t\right)
$$

and nonoscillation of (1.4) follows from Theorem 3.1 and considerations prior to (3.9).

Now we turn our attention to the second main result of the paper, (non)oscillation criteria for the perturbed generalized Riemann-Weber equation. In [2], influence of perturbations of the half-linear Riemann-Weber equation with critical coefficients

$$
\left(\Phi\left(x^{\prime}\right)\right)^{\prime}+\left(\frac{\gamma_{p}}{t^{p}}+\frac{\mu_{p}}{t^{p} \log ^{2} t}\right) \Phi(x)=0
$$

on its oscillatory behavior were investigated. It was shown $[2$, Corollary 1$]$ that the equation

$$
\left(\Phi\left(x^{\prime}\right)\right)^{\prime}+\left(\frac{\gamma_{p}}{t^{p}}+\frac{\mu_{p}}{t^{p} \log ^{2} t}+c(t)\right) \Phi(x)=0
$$

is oscillatory provided

$$
\int^{\infty} c(t) t^{p-1} \log t d t=\infty
$$

and it is nonoscillatory provided the integral $\int^{\infty} c(t) t^{p-1} \log t d t$ is convergent and

$$
\begin{aligned}
& \limsup _{t \rightarrow \infty} \log _{2}(t) \int_{t}^{\infty} c(s) s^{p-1} \log s d s<\mu_{p}, \\
& \liminf _{t \rightarrow \infty} \log _{2}(t) \int_{t}^{\infty} c(s) s^{p-1} \log s d s>-3 \mu_{p} .
\end{aligned}
$$

It was conjectured that under the assumption that $c(t) \geq 0$ for large $t$, equation (3.11) is oscillatory provided

$$
\liminf _{t \rightarrow \infty} \log _{2}(t) \int_{t}^{\infty} c(s) s^{p-1} \log s d s>\mu_{p}
$$

We will prove this conjecture in the general case, when oscillation of perturbed generalized half-linear Riemann-Weber equation with the critical coefficients

$$
\left(\Phi\left(x^{\prime}\right)\right)^{\prime}+\left(\frac{\gamma_{p}}{t^{p}}+\sum_{j=1}^{n} \frac{\mu_{p}}{t^{p} \log _{j}^{2}(t)}+c(t)\right) \Phi(x)=0
$$

is investigated. 
Theorem 3.3. Suppose that the integral $\int^{\infty} c(t) t^{p-1} \log _{n}(t) d t$ is convergent.

(i) If

$$
\begin{aligned}
& \limsup _{t \rightarrow \infty} \log _{n+1}(t) \int_{t}^{\infty} c(s) s^{p-1} \log _{n}(s) d s<\mu_{p}, \\
& \liminf _{t \rightarrow \infty} \log _{n+1}(t) \int_{t}^{\infty} c(s) s^{p-1} \log _{n}(s) d s>-3 \mu_{p},
\end{aligned}
$$

then equation (3.13) is nonoscillatory.

(ii) Suppose that there exists a constant $\gamma>\frac{2 \gamma_{p} p(p-2)}{3(p-1)^{2}}$ such that

$$
c(t) t^{p} \log ^{3} t \geq \gamma \quad \text { for large } t .
$$

If

$$
\liminf _{t \rightarrow \infty} \log _{n+1}(t) \int_{t}^{\infty} c(s) s^{p-1} \log _{n}(s) d s>\mu_{p},
$$

then (3.13) is oscillatory.

Proof. We again use the modified Riccati substitution $v=h^{p} w-G$ with $h(t)=t^{(p-1) / p} \log _{n}(t)$. In addition to the computation in the proof of Theorem 3.2, we will also compute explicitly the coefficient by $\log ^{-3} t$ in the formula for $h(t) L_{E}(h(t))$. We have for

$$
B:=\left(1+\frac{1}{(p-1) \log t}+\frac{1}{(p-1) \log _{2}(t)}+\cdots+\frac{1}{(p-1) \log _{n-1}(t)}\right)^{p-2}
$$

the expansion

$$
\begin{aligned}
B= & +\frac{p-2}{p-1}\left(\frac{1}{\log t}+\cdots+\frac{1}{\log _{n-1}(t)}\right) \\
& +\frac{(p-2)(p-3)}{2(p-1)^{2}}\left(\frac{1}{\log t}+\cdots+\frac{1}{\log _{n-1}(t)}\right)^{2}+ \\
& +\frac{(p-2)(p-3)(p-4)}{6(p-1)^{3}}\left(\frac{1}{\log t}+\cdots+\frac{1}{\log _{n}(t)}\right)^{3}+o\left(\log ^{-3} t\right)
\end{aligned}
$$

as $t \rightarrow \infty$. Multiplying this expansion by the expression $A$ from the proof of Theorem 3.2 we find that the coefficient by $\log ^{-3} t$ is

$$
\gamma_{p}\left[-\frac{(p-2)(p-3)(p-4)}{6(p-1)^{3}}+\frac{(p-2)^{2}(p-3)}{2(p-1)^{3}}-\frac{p-2}{p-1}\right]=\frac{2 \gamma_{p} p(2-p)}{3(p-1)^{2}} .
$$

Denote

$$
L_{R W}(x):=\left(\Phi\left(x^{\prime}\right)\right)^{\prime}+\left[\frac{\gamma_{p}}{t^{p}}+\sum_{j=1}^{n} \frac{\mu_{p}}{\log _{j}^{2}(t)}+c(t)\right] \Phi(x) .
$$

Then

$$
\begin{aligned}
h(t) L_{R W}(h(t)) & =\frac{2 \gamma_{p} p(2-p)}{3(p-1)^{2}} \frac{\log _{n}(t)}{t \log ^{3}(t)}+c(t) t^{p-1} \log _{n}(t)+o\left(\log _{n}(t) /\left(t \log ^{3} t\right)\right) \\
& =\frac{\log _{n}(t)}{t \log ^{3} t}\left[\frac{2 \gamma_{p} p(2-p)}{3(p-1)^{2}}+c(t) t^{p} \log ^{3} t+o(1)\right] .
\end{aligned}
$$


In part (ii) of Theorem 3.1 we need no sign restriction on the function $h[L(h)+\hat{L}(h)]$ which equals $h L_{R W}(h)$ in our case. Similarly as in the proof of Theorem 3.2

$$
\int^{t} R^{-1}=(1+o(1))\left(\frac{p-1}{p}\right)^{2-p} \log _{n+1}(t)
$$

and

$$
\lim _{t \rightarrow \infty} \log _{n+1}(t) \int_{t}^{\infty} \frac{\log _{n}(t)}{t \log ^{3} t}=0 .
$$

Then (3.14) is rewritten as (3.6). In the oscillation part (i) of Theorem 3.1 we need $h[L(h)+$ $\hat{L}(h)] \geq 0$ for large $t$ which leads to restrictions (3.15). Formula (3.16) is then rewritten as formula (3.5).

\section{Remarks and comments}

(i) Perturbations of the critical Euler equation

$$
\left(\Phi\left(x^{\prime}\right)\right)^{\prime}+\frac{\gamma_{p}}{t^{p}} \Phi(x)=0
$$

investigated in our paper contain iterated logarithms as appeared in (1.4). A natural question is whether one can investigate also other perturbations which "match together" similarly as the pairs $\frac{\alpha_{j}}{\log _{j}^{2}(t)}$ and $\frac{\beta_{j}}{t^{p} \log _{j}^{2}(t)}$ in (1.4). Consider the equation

$$
\left[(1+\lambda \alpha(t)) \Phi\left(x^{\prime}\right)\right]^{\prime}+\left[\frac{\gamma_{p}}{t^{p}}+\mu \beta(t)\right] \Phi(x)=0,
$$

where $\lambda, \mu$ are real-valued parameters. The modified Riccati substitution (2.2) with $h(t)=$ $t^{(p-1) / p}$ applied to (4.1) yields the modified Riccati equation (as can be verified by a direct computation)

$$
v^{\prime}+\lambda \Gamma_{p} t^{\frac{p-1}{p}}\left(\alpha(t) t^{-\frac{p-1}{p}}\right)^{\prime}+\mu t^{p-1} \beta(t)+\frac{p-1}{t(1+\alpha(t))^{q-1}} H\left(v, \Gamma_{p}\right)=0 .
$$

Under the assumption $\alpha(t)=o(1)$ as $t \rightarrow \infty$ (in order to have the function $1+\lambda \alpha(t)$ positive for large $t$ for any $\lambda \in \mathbb{R}$ ) the limited expression in Theorem 3.1 is

$$
\log t \int_{t}^{\infty}\left[\lambda \Gamma_{p} s^{\frac{p-1}{p}}\left(\alpha(s) s^{-\frac{p-1}{p}}\right)^{\prime}+\mu s^{p-1} \beta(s)\right] d s .
$$

Consequently, if

$$
\begin{aligned}
\lim _{t \rightarrow \infty} \log t \int_{t}^{\infty} s^{\frac{p-1}{p}}\left(\alpha(s) s^{-\frac{p-1}{p}}\right)^{\prime} d s & =: L_{\alpha}, \\
\lim _{t \rightarrow \infty} \log t \int_{t}^{\infty} s^{p-1} \beta(s) d s & =: L_{\beta}
\end{aligned}
$$

exist finite, by Theorem 3.1 (non)oscillation of (4.1) depends on whether

$$
\lambda \Gamma_{p} L_{\alpha}+\mu L_{\beta} \gtrless \frac{1}{2 q} .
$$


(ii) In [4], a general approach to two-parametric conditional oscillation of half-linear equations was treated. More precisely, the half-linear equation

$$
\left[(r(t)+\lambda \hat{r}(t)) \Phi\left(x^{\prime}\right)\right]^{\prime}+[c(t)+\mu \hat{c}(t)] \Phi(x)=0
$$

was investigated as a perturbation of (1.1). It is shown that if $h$ is a positive solution of (1.1), then under (2.5) and (2.6) the pair $\hat{r}, \hat{c}$ form a matching pair (oscillation of (4.3) depends on the value of a linear combination of $\lambda, \mu)$ provided there exist limits (the second one being finite)

$$
\lim _{t \rightarrow \infty} \frac{r(t)\left|h^{\prime}(t)\right|^{p}}{c(t) h^{p}(t)}, \quad \lim _{t \rightarrow \infty} \frac{\left(\hat{r}(t) \Phi\left(f^{\prime}(t)\right)\right)^{\prime}}{\hat{c}(t) \Phi(f(t))}
$$

where $f(t)=h(t)\left(\int^{t} R^{-1}(s) d s\right)^{1 / p}$ with $R=r h^{2}\left|h^{\prime}\right|^{p-2}$. Using the transformation approach we can suggest another condition for a matching pair, namely the existence of the finite limits

$$
\begin{aligned}
& \lim _{t \rightarrow \infty}\left(\int^{t} R^{-1}(s) d s\right)\left(\int_{t}^{\infty} h(s)\left(\hat{r}(s) \Phi\left(h^{\prime}(s)\right)\right)^{\prime} d s\right), \\
& \lim _{t \rightarrow \infty}\left(\int^{t} R^{-1}(s) d s\right)\left(\int_{t}^{\infty} h^{p}(s) \hat{c}(s) d s\right) .
\end{aligned}
$$

(iii) We have used in Theorem 3.3 as a transformation function in the modified Riccati substitution the function $h(t)=t^{(p-1) / p} \log _{n}^{1 / p}(t)$. This function asymptotically approximates the so-called principal solution of $(3.13)$ with $c(t) \equiv 0$, see $[12,13]$. In [12], it is shown that nonprincipal solutions of this equation behave asymptotically as the function $\tilde{h}(t)=C t^{(p-1) / p} \log _{n}^{1 / p}(t) \log _{n+1}^{2 / p}(t), C \in \mathbb{R}$. This suggests to use this function in the modified Riccati substitution as well. This would lead to (non)oscillation criteria where the limited formulas in Theorem 3.3 are replaced by

$$
\frac{1}{\log _{n+1}(t)} \int^{t} c(s) s^{p-1} \log _{n}(s) \log _{n+1}^{2}(s) d s .
$$

This problem is a subject of the present investigation. Note that in case $n=0$ criteria of this kind are given in [10].

\section{Acknowledgement}

Research supported by the Grant 201/11/0768 of the Czech Grant Foundation.

\section{References}

[1] R. P. Agarwal, S. R. Grace, D. O'Regan, Oscillation theory of second order linear, halflinear, superlinear and sublinear dynamic equations, Kluwer Academic Publishers, Dordrecht/Boston/London, 2002. MR2091751

[2] O. DošLÝ, Perturbations of half-linear Euler-Weber differential equation, J. Math. Anal. Appl. 323(2006), 426-440. MR2662216; url 
[3] O. DošLÝ, S. FišNaRová, Half-linear oscillation criteria: perturbation in term involving derivative. Nonlinear Anal, 73(2010), No. 12, 3756-3766. MR2728552; url

[4] O. DošLÝ, S. FIŠnarová, Two-parametric conditionally oscillatory half-linear differential equations, Abstr. Appl. Anal. 2011, Art. ID 182827, 16 pp. MR2771214; url

[5] O. DošLÝ, S. FišNARová, Variational technique and principal solution in half-linear oscillation criteria, Appl. Math. Comput. 217(2011), No. 12, 5385-5391. MR2770115; url

[6] O. DošLÝ, H. Funková, Perturbations of half-linear Euler differential equation and transformations of modified Riccati equation, Abstr. Appl. Anal. 2012, Art. ID 738472, 19 pp. MR2991019; url

[7] O. Došlý, H. Haladová, Half-liner Euler differential equations in the critical case, Tatra Mt. Math. Publ. 48(2011), 41-49. MR2841104; url

[8] O. DošLx́, A. Lomtatidze, Oscillation and nonoscillation criteria for half-linear second order differential equations, Hiroshima Math. J. 36(2006), 203-219. MR2559737

[9] O. DošLÝ, P. К̌EHÁK, Half-linear differential equations, North-Holland Mathematics Studies, Vol. 202, Elsevier, 2005. MR2158903

[10] O. DošLÝ, J. ŘEzníčKová, Nonprincipal solutions in oscillation criteria for half-linear differential equations, Stud. Sci, Math. Hungar. 47(2010), 127-137. MR2654234; url

[11] Á. Elbert, Asymptotic behaviour of autonomous half-linear differential systems on the plane, Studia Sci. Math. Hungar. 19(1984), 447-464. MR0874513

[12] Á. Elbert, A. Schneider, Perturbations of the half-linear Euler differential equation, Result. Math. 37(2000), 56-83. MR1742294; url

[13] Á. Elbert, T. Kusano, Principal solutions of nonoscillatory half-linear differential equations, Adv. Math. Sci. Appl. 18(1998), 745-759. MR1657164

[14] P. Hartman, Ordinary differential equations, Birkhäuser, Boston, 1982. MR0658490

[15] P. Hasil, Conditional oscillation of half-linear differential equations with periodic coefficients, Arch. Math. (Brno) 44(2008), 119-131. MR2342849

[16] H. KRÜGer, G. Teschl, Effective Prüfer angles and relative oscillation criteria, J. Differential Equations 245(2009), 3823-3848. MR2462706; url

[17] T. Kusano, J. Manojlović, T. Tanigawa, Comparison theorems for perturbed half-linear Euler differential equations, Int. J. Appl. Math. Stat. 9(2007), No. J07, 77-94. MR2339349 